\title{
Empirical Mode Decomposition for Speech Synthesis
}

\author{
Ram Kumar Panthi ${ }^{1}$, Prof. Jyotsna Ogale ${ }^{2}$ \\ M. Tech, (EC) SATI, Vidisha ${ }^{1}$ \\ Associate Prof, EC Department SATI, vidisha ${ }^{2}$
}

\begin{abstract}
A new method of Synthesis by Analysis for EMD multi-component signals of fast changing instantaneous attributes is introduced. It makes use of two recent developments for signal decomposition to obtain near monocomponent signals whose instantaneous attributes can be used for synthesis. Furthermore, by extension and combination of both decomposition methods, the overall quality of the decomposition is shown to improve considerably summarize the use of Empirical mode decomposition (EMD) for denoising a speech signal. EMD, introduced by Huang et al in gives time-frequency representation of non-linear and non-stationary signals. It decomposes a signal into a sum of finite zero-mean, oscillating components called as intrinsic mode functions based on the local time characteristics of the signal. Main essence of the method is it's adaptive and data driven nature.
\end{abstract}

Keywords: Empirical mode decomposition, IMF, Hilbert transform, Denoising technique.

\section{INTRODUCTION}

The synthesis by analysis approach for sounds with fast changing attributes still poses problems. These can be traced back up to a large degree to the fundamental mathematical properties of the underlying TimeFrequency (TF) analysis methods. This paper introduces two recent methods for wide-band signal decomposition in the context of audio analysis and synthesis for such problematic signals. The requirement is that the components contained are sufficiently spaced apart in the spectrum. Similar to the spectral modeling synthesis introduced by Serra [1], the analysis decomposes a given signal into a sum of time-varying sinusoids plus residual. Here, stochastic components are split into frequency bands and not necessarily part of the residual. The precision of instantaneous phase information obtained by the analysis facilitates phase alignment for the synthesis, thus transients can be retained. Additionally, the involved decomposition methods eliminate the need to perform peak-continuation of spectral components. First, the analysis which is intended to be performed offline is shown, section 3 shows the method of resynthesis which can be performed online. Finally, the paper concludes with results on the Quality of the method and gives future directions for improvement.

\section{ANALYSIS}

Time-Frequency representations give insight into the complex structure of time series signals by revealing their comprising components within Temporal and spectral localization. The majority of algorithms performing such a representation on multi-component signals consist roughly of linear And quadratic ones. Representatives for the first group are the Short-Time Fourier and Wavelet Transformations and, respectively, the Wigner-Ville
Distribution for the latter one. The first group relies on the linear super-position Principle of base functions with which the signal to be analyzed is compared [2]. As such a basis is chosen a priori, presumptions are made in regards to the driving mechanisms of the data. In consequence, misfits in respect to the selected basis are assigned to various orders of harmonics thereof, thus coloring or possibly depriving the TF representation of physical meaning, especially if the data is the non-stationary result of non-linear driving mechanisms. Besides this, such integral transforms obey the Heisenberg-Gabor limit, forcing a trade-off for either time or frequency localization. Quadratic methods, on the other hand, avoid the use of basic functions as templates and generally provide a high-resolution $\mathrm{TF}$ representation for monocomponent signals (defined below). However, for multicomponent signals, the additional presence of interference terms between each pair of individual components can severely distort the representation. Removing them by means of filtering comes at the expense of TF resolution.

Alternatively, a signal can be regarded as the result of superimposed mono-components. A mono-component is a sinusoid whose attributes are instantaneous - amplitude and phase vary with time. It exhibits a well-behaved Hilbert-Transform (HT), so the derived analytic signal reflects these attributes uniquely and unambiguously. The question is, within the infinite possibilities to decompose a signal, how can multi-component signals be separated into such mono-components? In the last decade mainly two approaches towards this have emerged: the Empirical Mode Decomposition (EMD) [3] and the Hilbert Vibration Decomposition (HVD) [4]. Both are nonparametric and adaptive decompositions with base functions chosen a posterior. The reason they will be shown in a little bit 
Vol. 5, Issue 12, December 2016

more detail is that the proposed method makes use of both of them in a way to diminish their mutual downsides.

\section{INSTANTANEOUS ATTRIBUTES OF MONO- COMPONENTS}

One way to obtain the instantaneous attributes of a monocomponent signal $x(t)$, the amplitude $A(t)$ and the phase $(\mathrm{t})$, is by constructing its complex valued analytic signal $\mathrm{X}(\mathrm{t})$. This can be achieved by composing the original time-domain signal $x(t)$ with its imaginary HilbertTransformed version $\dot{x}(t)$ (the quadrature projection). As a result, the

Instantaneous amplitude and phase can be determined

$$
\begin{gathered}
\text { As } \sqrt{\mathrm{x}^{2}(\mathrm{t})+\dot{\mathrm{x}}^{2}(\mathrm{t})}=\mathrm{A}(\mathrm{t}), \\
\Phi(\mathrm{t})=\arctan \frac{\dot{\mathrm{x}}(\mathrm{t})}{\mathrm{x}(\mathrm{t})}
\end{gathered}
$$

Throughout the rest of the paper $\Phi(t)$ denotes the unwrapped instantaneous phase function.

\section{Empirical Mode Decomposition}

Empirical mode decomposition (EMD) decomposes a signal $x(t)$ into a finite number of Intrinsic Mode Functions (IMFs),

$$
\begin{array}{r}
h(i)(t), 1 \leq i \leq L, \\
x(t)=\sum_{i=1}^{L} h_{i}(t)+r(t)
\end{array}
$$

Where $r(t)$ is a remainder which is a non zero-mean slowly varying function with only few extreme. Decomposition is based on the characteristics of the signal itself. IMFs are zero-mean oscillating signals satisfying the following conditions:

A. The number of extreme and the number of zero crossings must either be equal or differ at most by one,

B. At any point, the mean value of the envelope defined by local maxima and the envelope defined by the local minima is zero. Steps for finding the IMFs of a signal are as follows-

- Identify local maxima and minima of $x(t)$.

- 2) Form the upper and lower envelope $u(t)$ and $1(t)$ by cubic spline interpolation of the extrema points.

- 3) Calculate the mean of the upper and lower envelop, $\mathrm{m} 1(\mathrm{t}) \mathrm{u} \operatorname{sing} \mathrm{m} 1(\mathrm{t})=\mathrm{u}(\mathrm{t})+\mathrm{l}(\mathrm{t}) / 2$.

- 4) Subtract mean from the signal $x(t)$ to obtain $d 1(t)$. If $\mathrm{d} 1(\mathrm{t})$ is a zero-mean function, then the iteration stops and $\mathrm{d} 1(\mathrm{t})$ is accepted as first IMF,ie $\mathrm{h} 1(\mathrm{t})=\mathrm{d} 1(\mathrm{t})$.

- 5) If not, use $\mathrm{d} 1(\mathrm{t})$ as the new data and repeat steps 1-4 until an IMF is obtained.

- 6) Once the first IMF h1(t) is obtained, residual signa is defined as

$$
\mathrm{r} 1(\mathrm{t})=\mathrm{x}(\mathrm{t})-\mathrm{h} 1(\mathrm{t})
$$

Residual signal contains information about the lower A2 create interplant e min(t) through the local minima and frequency components and is taken as the input signal to respectively e max(t) through the local maxima A3 set obtain next IMFs. At the end, a monotonic function with $m(t)$ as local average with only few extreme is obtained from which no further decomposition can be done.

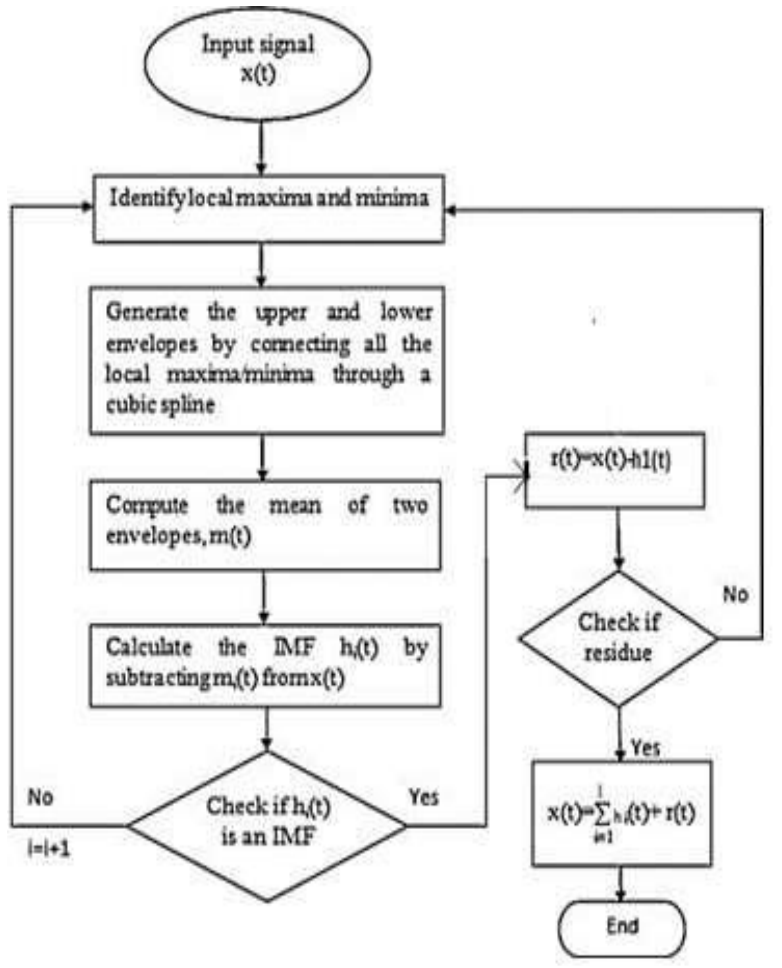

Fig 3.1Flow chart

\section{CASE STUDY}

At the core of the EMD is a sifting process that creates almost mono-components [4]. The sifting is performed by identifying the innate undulations belonging to different relative frequency scales and recursively discerning waves riding on top of each other using repeated approximation. By means of this scale separation, intrinsic modes of oscillations are extracted from signal $\mathrm{s}(\mathrm{t})$. These are called intrinsic mode functions (IMFs), hk(t), if they fulfil: (a) for $\mathrm{hk}(\mathrm{t})$ the number of extreme points $(\mathrm{min} / \mathrm{max})$ and zero crossings are equal or differ at most by one (b) the mean of the lower envelope defined by the local minima and the respective upper envelope of $\mathrm{hk}(\mathrm{t})$ is at any point zero With the global residue (or trend) $\mathrm{r}(\mathrm{t}), \mathrm{s}(\mathrm{t})$ can be expressed as:

$$
\sum_{k=1}^{n} h k+r(t)=\mathrm{Ak}(\mathrm{t}) \cos \_\mathrm{k}(\mathrm{t})+\mathrm{r}(\mathrm{t})
$$

Where $\mathrm{n}$ is the number of IMFs extracted. As equation 2 suggests, an IMF has variable amplitude and frequency as functions of time and therefore constitutes the opposite of mono-harmonic signal. Figure 1 exemplifies such decomposition. The objective to find IMFs is performed by a sifting process, starting with

$$
\mathrm{r}(\mathrm{t})=\mathrm{rp}(\mathrm{t})=\mathrm{s}(\mathrm{t}) \text { and } \mathrm{i}=\mathrm{k}=0 \text { : }
$$


$\mathrm{m}(\mathrm{t})=\frac{E \min +E \max }{2} \quad \mathrm{~A} 4$

Define a "proto-mode" function

$$
\operatorname{pi}(\mathrm{t})=\operatorname{rp}(\mathrm{t})-\mathrm{m}(\mathrm{t}),
$$

Set $\operatorname{rp}(\mathrm{t})=\operatorname{pi}(\mathrm{t})$ and $\mathrm{i}=\mathrm{i}+1$

A5 repeat steps A1-4 until pi(t) meets stopping criterion $S$; then an IMF is found,

$$
\operatorname{hk}(\mathrm{t})=\operatorname{pi}(\mathrm{t})
$$

A6 set $\mathrm{r}(\mathrm{t})=\mathrm{r}(\mathrm{t})-\mathrm{hk}(\mathrm{t})$; if stopping criterion $\mathrm{T}$ is fulfilled then terminate, else $\mathrm{i}=0, \mathrm{k}=\mathrm{k}+1$ and $\mathrm{rp}(\mathrm{t})=\mathrm{r}(\mathrm{t})$; restart from step A1. Here, steps A1-4 create a k-level IMF and step A5 controls the global sifting process. In this way, the EMD repeatedly removes a wave riding on top of the local residue $\mathrm{r}(\mathrm{t})$ as it identifies the wave through local extreme points and treats the residue at each Level as global trend. At the whole, the behaviour of the EMD is similar toa filter-bank: performing as high-pass filter for the first IMF and as band-pass for successive IMFs. Yet the characteristic is that the cut-off/centre frequencies are nonstationary. Albeit the EMD is still of algorithmic nature, some theoretical work has been put introit to describe its behaviour. When analyzing white noise-like wideband signals, the EMD behaves like a dyadic filter-bank [5], while for bi component signals of harmonics there exists a theoretical limit for separation of

$$
\mathrm{A} 1 / \mathrm{A} 2=(\mathrm{F} 1 / \mathrm{F} 2)^{2}=1[6] .
$$

Hence, the EMD does not perform well when the components' frequencies are close or differ little in amplitude. The existence of a plethora of implementations for the EMD make further theoretical assessment difficult as some tackle core issues of the algorithm like the choice of the interpolation technique or the construction of the envelope differently. As suggested by Huang [3], the cubic spine interpolation is Used here. The condition criteria for the envelope are currently not completely understood [7], leading to various contributions how extreme points ought to be chosen. Instead of finding the local extreme of $s(t)$ itself, it is proposed to find them in the inverse of the second derivative of $\mathrm{s}(\mathrm{t})$. Hence, first the "frequency resolution" is increased for riding waves that are partially immersed in the local trend and thus do not produce local extrema (e.g saddle points), and second, for pure sinusoids the positions stay the same. This approach, however, comes at the danger of producing artificial vibrations, especially in lower IMFs. Consequently it is applied for the first IMFs only $(\mathrm{k} \leq 5)$ where most of the high frequency contents of $\mathrm{s}(\mathrm{t})$ are to be expected. In figure 2 an example Is given where this method helps to uncover positions of extreme. Regarding the stopping criteria: for the number of IMFs generated can be either set to a fixed amount of iterations, commonly $\mathrm{k}_{-} \log 2 \mathrm{~N}$ with $\mathrm{N}$ being the number of data points of $\mathrm{s}(\mathrm{t})$, or an indicator that the residue still contains oscillations. Here, the former criterium was applied as for the used test signal $\left(\mathrm{N}_{-}\right.$
22050), the final residue always showed a non-oscillatory trend. For $\mathrm{S}$ a number of stopping criteria have been suggested, the original recommendation being to set the number of iterations to the order of tens. Accordingly, the number of iterations was set to $i=30$. However, they are terminated before the cubic spines interpolation leads to degenerated results. This condition is met as soon as the area under the cubic splines increases in succeeding iterations $i$. This is an indicator for large overshoots of the interpolation caused by ill-conditioned extrema points. Thus, the possibility of degenerated envelopes creating artificial vibrations for succeeding IMFs is reduced. A major problem that exists for the EMD is the phenomenon of mode-mixing that results in a) an IMF containing signals of widely disparate scales or b) signals of similar scale residing in different IMF components [7]. This happens when the intermittency in the extrema detected belongs to different signals as caused when parts of the riding wave are completely immersed in the local trend. Several methods have been proposed to alleviate this problem; commonly the aim is to emphasize "lost" extrema points of the riding Wave. In general, there are two approaches to this: either calculates the mean of an ensemble of decompositions that have different instances Of noise added to the signal (EEMD) [8], or add masking signals in the decomposition that approximate the riding waves in the problematic areas [7,9]. Initial attempts to use the EEMD resulted in less mode mixing of type b) but more of type a), when components reside closely in a frequency band with similar amplitudes. Therefore, the use of masking signals has been chosen. Suppose that a masking signal $\wedge^{\wedge} \mathrm{r}(\mathrm{t})$ that contains For the sake of brevity, the HVD is only superficially presented here. As opposed to the EMD, the HVD is entirely based on the HT. Therefore, the HVD does not depend on a dissimilar harmonics amplitude ratio as does the EMD. The method is based on the observation that, in a multi-component signal, the instantaneous attributes of the component with the highest energy change more slowly in comparison to the sum of those of the underlying components.

In order to rid a signal of these fast oscillating instantaneous attributes and thereby performing the decomposition, the instantaneous attributes derived by means of the HT are low-pass filtered. The filtered result is seen to constitute a mono-component. The residue can again be used in the decomposition process leading to a set of basic functions that similarly express $s(t)$ as in equation 2. By applying the HVD for only one iteration (to obtain the singular highest energy component) on $r(t)$ from the EMD, the masking signal $\wedge$ is generated. The reason the HVD is not used principally for the decomposition is that the HT is very sensitive to false spikes or random noise that leads to the distortion of transients in the instantaneous attributes or smearing [4]. The EMD, on the other hand, is capable of decomposing noisy signals [5]. Also, due to practical limitations of precise low-pass filtering in the HVD, the number of extracted components is limited [4]. However, in general the HVD is able to 
Vol. 5, Issue 12, December 2016

better separate components in a narrow band than the resynthesis expectedly introduced perceivable glissandi EMD. By combining both methods this way the HVD around such sections of mode-mixing. When disregarding helps increasing the frequency resolution of the EMD and these sections the results were satisfactory as the timbre of reducing mode-mixing errors. To compare the the sound was preserved (1octave, $2 \mathrm{x}$ time-stretches) once performance of this approach to the original EMD one, the the post-processing removed the unwanted modulations. quality of the decomposition of a bi-component signal was Since the EMD is able to decompose noisy signals, the measured in the same way as discussed in [6]. Due to the sample of a (real) snare could be decomposed into separate Recursive nature of the EMD such comparison gives IMFs containing noise (dyadic frequency bands) and a insight into the overall decomposition performance for tonal component.

complex multi-component signals. Figure 3 allows the comparison of the ability of both methods to identify a Similarly, the sample of a (real) cowbell was successfully high frequency signal $\mathrm{xh}(\mathrm{t})$ within a composition $\mathrm{x}(\mathrm{t})$ of decomposed into fundamental and harmonics. For all of $\mathrm{xh}(\mathrm{t})$ and a low frequency signal $\mathrm{xl}(\mathrm{t})$. As can be seen in these percussive samples, the fundamental could be well plot 3 a), the proposed

\section{EXPERIMENTAL RESULT}

The quality of the resynthesis depends very much on the effectiveness of the post-processing and the presence of mode-mixing in the obtained components. For example, the resynthesis of a monophonic (synthetic) bass-drum (cp. table 1) without post-processing led to a change of the originally sinusoidal signal to a more square wave-like one due to the errors introduced by the Hilbert FIR. With postprocessing, the resynthesized audio had no perceivable differences.

For the piano sample, the decomposition introduced mode mixing errors in the decay phase of the sound as extreme of the previously correctly tracked high-frequency were immersed in lower frequency removing low-energy modulations of phase and amplitude. harmonics. This resulted in perceivable phase distortions Finally, a rough summary of the quality of the synthesized (bursts) when pitch-shifting or time-stretching; on sounds has been given.

reducing the value of the post-processing coefficients the

Table1 parameters of original signals before processing and after processing synthesized signals

\begin{tabular}{|l|c|c|c|c|c|c|}
\hline signals & $\begin{array}{c}\text { Standerd } \\
\text { deviation of } \\
\text { original } \\
\text { signal }\end{array}$ & $\begin{array}{c}\text { Standerd } \\
\text { deviation of } \\
\text { reconstructed } \\
\text { signal }\end{array}$ & $\begin{array}{c}\text { Mean of } \\
\text { original } \\
\text { signal }\end{array}$ & $\begin{array}{c}\text { Mean of } \\
\text { reconstructed } \\
\text { signal }\end{array}$ & $\begin{array}{c}\text { MSE between } \\
\text { original and } \\
\text { reconstructed } \\
\text { signal }\end{array}$ & $\begin{array}{c}\text { MSE between } \\
\text { power spectrum of } \\
\text { original and } \\
\text { reconstructed signal }\end{array}$ \\
\hline Bass drum & 0.5666 & 0.6170 & 0.1225 & 0.0445 & $6.5766 \mathrm{e}-007$ & $5.0380 \mathrm{e}-007$ \\
\hline bell & 0.5666 & 0.6170 & 0.1225 & 0.0445 & $6.5766 \mathrm{e}-007$ & $5.0380 \mathrm{e}-007$ \\
\hline paino & 0.5666 & 0.6170 & 0.1225 & 0.0445 & $6.5766 \mathrm{e}-007$ & $5.0380 \mathrm{e}-007$ \\
\hline snare & 0.5666 & 0.6170 & 0.1225 & 0.0445 & $6.5766 \mathrm{e}-007$ & $5.0380 \mathrm{e}-007$ \\
\hline
\end{tabular}

Table2 intrinsic mode function (imf) of signals \& noise reduction coefficients

\begin{tabular}{|c|c|c|c|c|c|c|c|c|c|c|c|c|c|c|}
\hline Signals & $\mathrm{nr}$ & $\mathrm{nc}$ & Imf1 & Imf2 & Imf3 & Imf4 & Imf5 & Imf6 & Imf7 & Imf8 & nrc & ncc & nrcp & nccp \\
\hline $\begin{array}{c}\text { Bass } \\
\text { drum }\end{array}$ & 8 & 65528 & 65528 & 65528 & 65528 & 65528 & 65528 & 65528 & 65528 & 65528 & 8 & 8883 & 8 & 8883 \\
\hline bell & 8 & 65528 & 65528 & 65528 & 65528 & 65528 & 65528 & 65528 & 65528 & 65528 & 8 & 8883 & 8 & 8883 \\
\hline paino & 8 & 65528 & 65528 & 65528 & 65528 & 65528 & 65528 & 65528 & 65528 & 65528 & 8 & 8883 & 8 & 8883 \\
\hline snare & 8 & 65528 & 65528 & 65528 & 65528 & 65528 & 65528 & 65528 & 65528 & 65528 & 8 & 8883 & 8 & 8883 \\
\hline
\end{tabular}


VI.RESULT

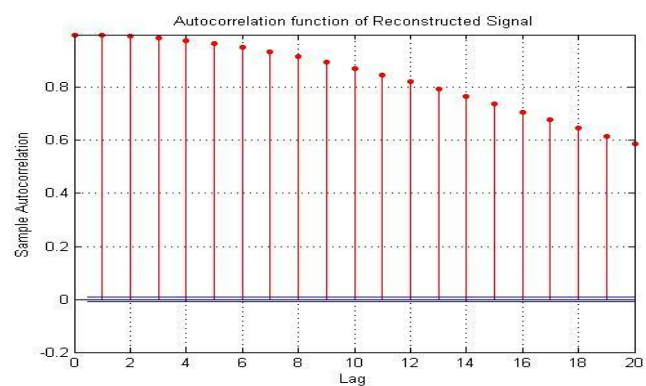

Fig.1autocorrelation function of original signal

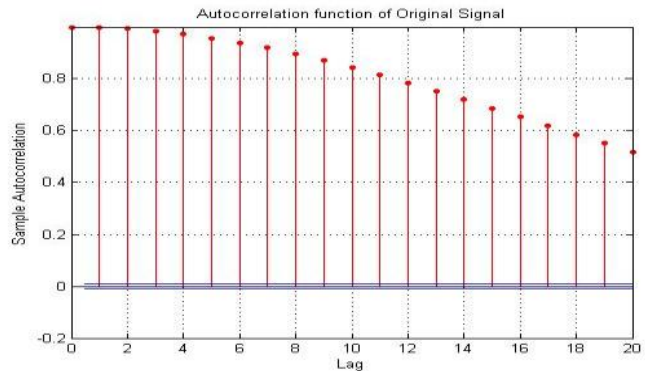

Fig.2 autocorrelation function of reconstructed signal

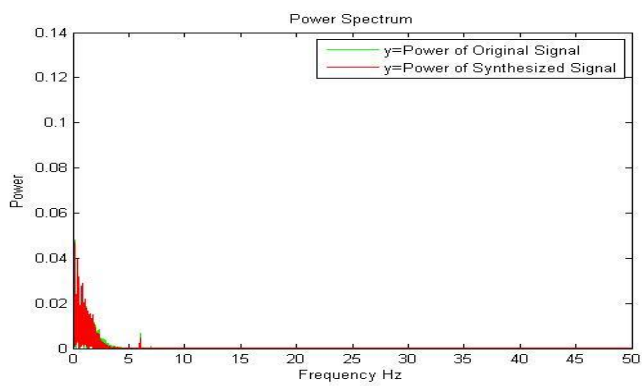

fig 3 power specterm

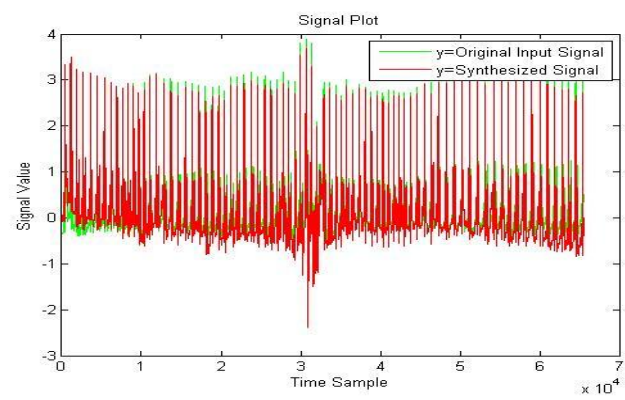

Fig.4 signal plot

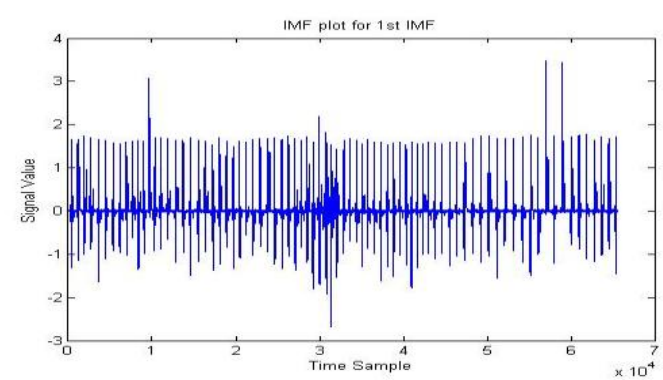

Fig.5IMF plot for $1^{\text {st }}$ IMF

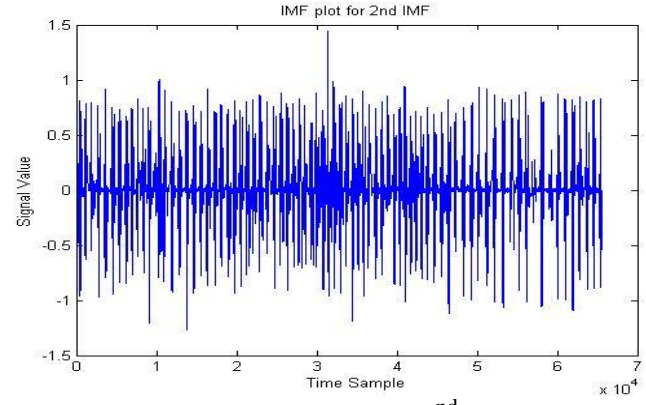

Fig.6 IMF plot for $2^{\text {nd }}$ IMF

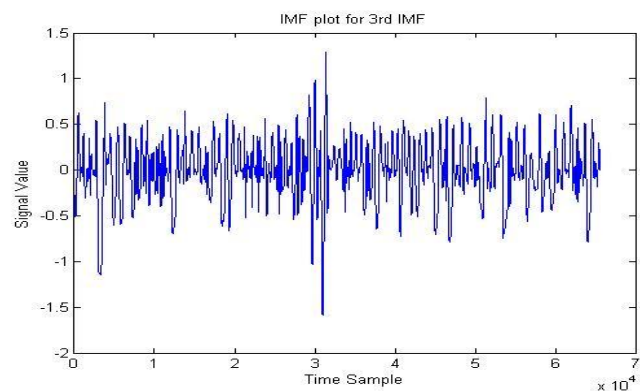

Fig.7 IMF plot for $3^{\text {rd }}$ IMF

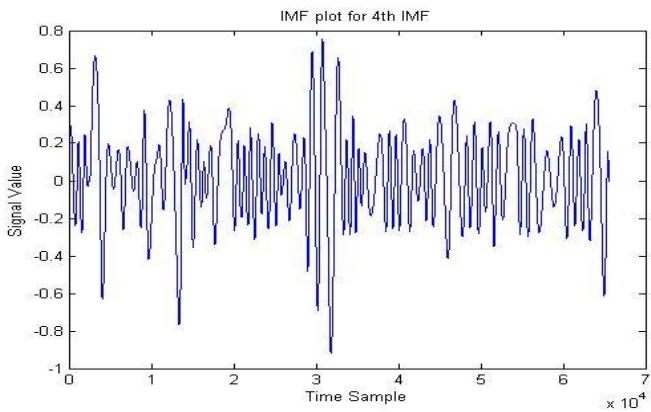

Fig.8 IMF plot for $4^{\text {th }}$ IMF

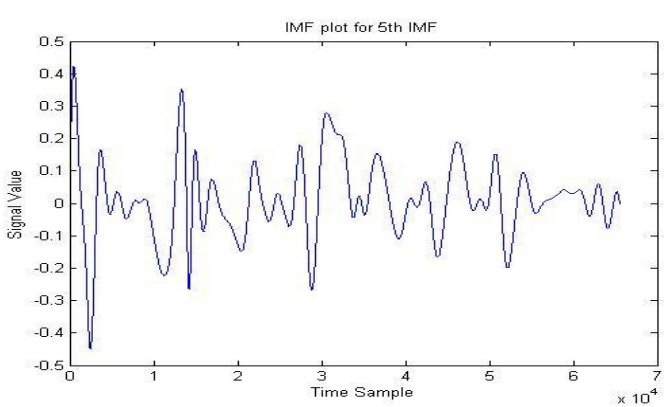

Fig.9 IMF plot for $5^{\text {th }}$ IMF

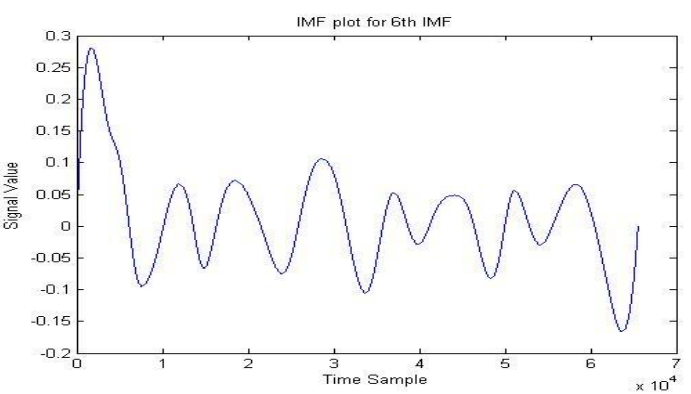

Fig.10 IMF plot for $6^{\text {th }}$ IMF 
Vol. 5, Issue 12, December 2016

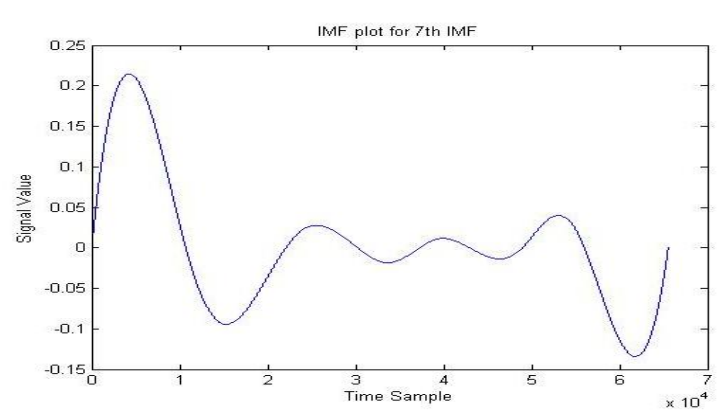

Fig. 11 IMF plot for $7^{\text {th }}$ IMF

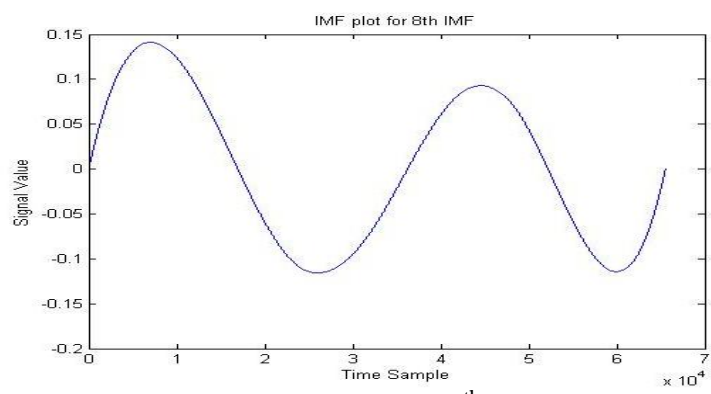

Fig,12IMF plot for $8^{\text {th }}$ IMF

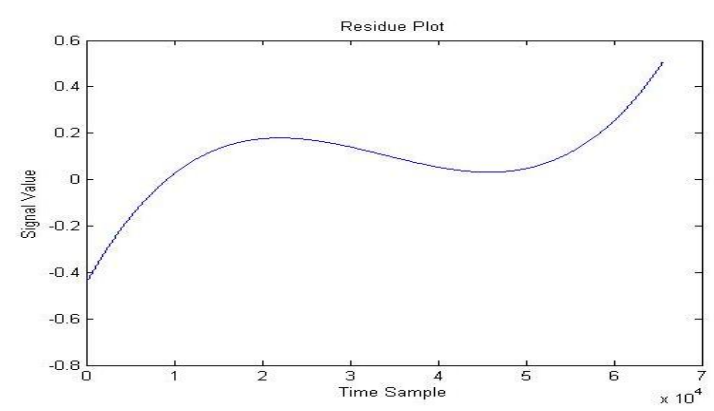

Fig.13 residue plot

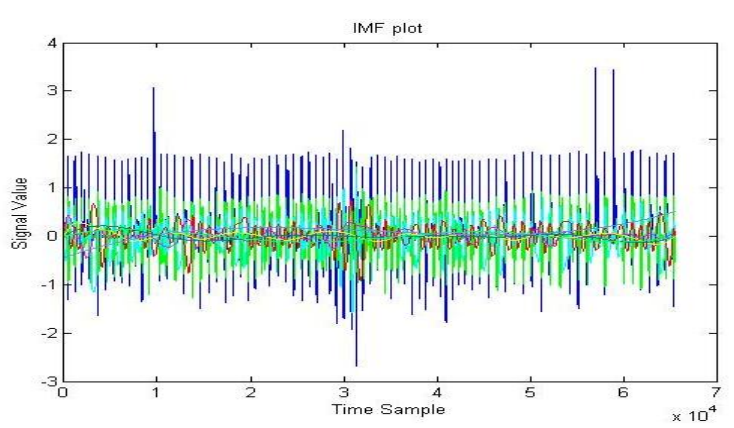

Fig.14IMF plot

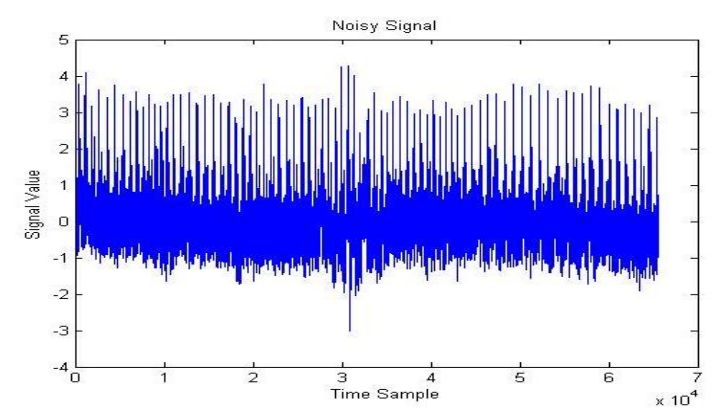

Fig.15 noisy signal

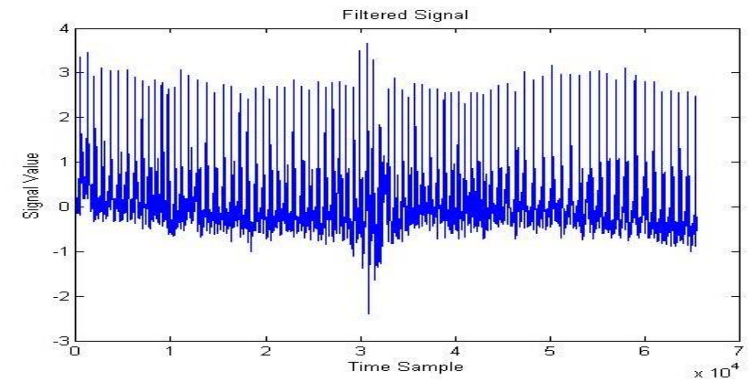

Fig .16 filtered signal

- Standard Deviation of Original Signal $=0.5666$

- Standard Deviation of Reconstructed Signal=0.6170

- Mean of Original Signal=0.1225

- Mean of Reconstructed Signal=0.0445

- Mean Square Error between Original and Reconstructed Signal $=6.5766 \mathrm{e}-007$

- Mean Square Error between Power Spectrum of Original and Reconstructed Signal $=5.0380 \mathrm{e}-007$

\section{CONCLUSION}

Hence, to conclude, the shown approach can be very well used to EMD wide-band signals with partials that have fast changing instantaneous attributes and are sufficiently spaced apart in the spectrum. An improvement to this approach would be to add a dedicated noise model to the sinusoidal one in order to be able to alter the behavior of noisy partials properly. As in the case of EMD a piano sound, there are remaining problems regarding the quality of the decomposition, most importantly the frequency and amplitude resolution. However, this may change with future developments of the EMD and To confirm the effectiveness of the method, a male speech is recorded and taken as the original signal. White Gaussian noise is used to model the background noise. Noisy speech signal is applied to the EMD algorithm taken from The obtained IMFs are filter using the principle of soft filter to recover an estimate of the original signal. al filter rule is used. All the simulations are done in MATLAB environment

\section{REFERENCES}

[1] X Serra and J Smith, "Spectral Modeling Synthesis: A Sound Analysis/Synthesis System based on a Deterministic plus Stochastic Decomposition," Computer Music Journal, vol. 14, no. 4, pp. 1224, 1990.

[2] Franz Hlawatsch and G. Faye Boudreaux-Bartels, "Linear and Quadratic Time Frequency Signal Representations," IEEE Signal Processing Magazine, vol. 9, no. 2, pp. 21 67, 1992.

[3] Norden E. Huang, Zhaohua Wu, Steven R. Long, Kenneth C. Arnold, Xianyao Chen, and Karin Blank, "ON INSTANTANEOUS FREQUENCY," Advances in Adaptive Data Analysis, vol. 1, no. 2, pp. 177-229, Dec. 2009.

[4] Michael Feldmann, Hilbert Transform Applications in Mechanical Vibration, Hoboken, N.J.:Wiley, 1 edition, 2011.

[5] P. Flandrin, G. Rilling, and P. Goncalves, "Empirical Mode Decomposition as a Filter Bank," IEEE Signal Processing Letters, vol. 11, no. 2, pp. 112-114, Feb. 2004.

[6] Gabriel Rilling and Patrick Flandrin, "One or two frequencies? The empirical mode decomposition answers," Signal Processing, IEEE Transactions, vol. 56, no. 1, pp. 85-95, 2008. 
[7] Xiyuan Hu, Silong Peng, and Wen-liang Hwang, "EMD Revisited : A New Understanding of the Envelope and Resolving the Mode Mixing Problem in AM-FM Signals," Agenda, , no. June, 2011.

[8] Zhaohua $\mathrm{Wu}$ and Norden E Huang, "Ensemble empirical mode decomposition for high frequency ECG noise reduction.," Advances in Adaptive Data Analysis, vol. 55, no. 4, pp. 193-201, 2009.

[9] Ryan Deering and James F Kaiser, "The use of a masking signal to improve empirical mode decomposition," Time, vol. 4, no. January, pp. 485-488, 2005.DAFX-4

[10] Y. Zhang, Y. Gao, L. Wang, J. Chen, and X. Shi, "The removal of wall components in doppler ultrasound signals by using the empirical mode decomposition algorithm," IEEE Trans. Biomed. Eng., vol. 9, pp. 1631-1642, Sept. 2007.

[11] L. Hadjileontiadis, "Empirical mode decompostion and fractal dimension filter," IEEE Eng. Med. Biol. Mag., pp. 30 - 39, Jan. 2007. [12] B. Ning, S. Qiyu, Y. Zhihua H. Daren, and H. Jiwu, "Robust image watermarking based on multiband wavelets and empirical mode decomposition," IEEE Trans. Image Processing, pp. 1956 - 1966, Aug. 2007.

[13] Md. K. I. Molla and K. Hirose, "Single-mixture audio source separation by subspace decomposition of hilbert spectrum," IEEE Trans. on Audio, Speech and Language Processing, pp. 893 - 900, Aug. 2007.

[14] S. Mallat, A wavelet tour of signal processing, Academic press, second edition, 1999.

[15] A. Antoniadis and J Bigot, "Wavelet estimators in nonparametric regression: A comparative simulation study," Journal of statistical software, vol. 6, pp. 1-83, 2001. [16] D. L. Donoho and I. M. Johnstone, "Ideal spatial adaptation by wavelet shrinkage," Biometrika, vol. 81, pp. 425-455, 1994.

[17] S. Theodoridis and K. Koutroumbas, Pattern Recognition, Academic press, third edition, 2006.

[18] H. C. Huang and N. Cressie, "Deterministic/stochastic wavelet decomposition for recovery of signal from noisy data," Technometrics, vol. 42, pp. 262-276, 2000.

[19] P. Flandrin, G. Rilling, and P. Gonc, alv`es, EMD equivalent filter banks, from interpetation to applications (in N. E. Huang and S. Shen, Hilbert-Huang Transform and Its Applications), World Scientific Publishing Company, first edition, 2005.

[20] Z. Wu and N. E. Huang, "A study of the characteristics of white noise using the empirical mode decomposition method," Proc. Roy. Soc. London A, vol. 460, pp. 1597-1611, June 2004.

[21] N. E. Huang Z. Wu, Statistical significance test of intrinsic mode functions, (in N. E. Huang and S. Shen, Hilbert-Huang Transform and Its Applications), World Scientific Publishing Company, first edition, 2005

[22] A. O. Boudraa and J. C. Cexus, "Denoising via empirical mode decomposition," in ISCCSP2006, 2006.

[23] Y. Mao and P. Que, "Noise suppression and flaw detection of ultrasonic signals via empirical mode decomposition," Russian Journal of Nondestructive Testing, vol. 43, pp. 196-203, 2007.

[24] T. Jing-tian, Z. Qing, T. Yan, L. Bin, and Z. Xiao-kai, "Hilberthuang transform for ECG de-noising," in 1st International Conference on Bioinformatics and Biomedical Engineering (ICBBE 2007), 2007. 